\title{
CT-Based Diagnosis of Occipital Condyle Fractures. A Prospective Evaluation With Reassessment of Fracture Morphology
}

\author{
Mueller Franz ${ }^{1 *}$, Rosskopf Michael ${ }^{1}$, Goessmann ${ }^{2}$, Holger $^{2}$ and Fuechtmeier Bernd ${ }^{1}$
}

${ }^{1}$ Clinic for Trauma and Orthopaedic Surgery, Barmherzige Brüder, Prufeninger, Germany

${ }^{2}$ University Regensburg, Institution for Radiological Diagnostic, Germany

\begin{abstract}
Background: CT analyses of occipital condyle fractures (OCF) have been based on low case numbers so far, and prospective studies on fracture morphology are not available yet.

Methods: Over a period of five years, all OCF were prospectively assessed, and the patients were followed-up clinically and radiologically after one year post trauma.

Results: 31 patients were assessed of whom 27 had unilateral OCFs and 4 had bilateral OCFs; a total of 5 patients $(16.1 \%$ ) with OCF died due to the severity of associated injuries. Coronary CT reconstructions either showed horizontal or vertical fracture lines, with the latter extending into the joint (14 versus 17 patients). Mortality was $37.5 \%$ in patients with horizontal fractures (due to associated cerebral injuries) and $0 \%$ in patients with vertical fractures with joint involvement. The outcome at the time of clinical follow-up was also significantly worse with horizontal fractures.

Conclusions: OCFs per se are generally considered to be benign injuries. However, fracture morphology is an indicator for the clinical course: Due to related severe intracerebral injuries, horizontal fractures - when compared to vertical fractures - are associated with higher morbidity, significantly higher mortality, and significantly worse outcome.
\end{abstract}

Keywords: Computed tomography; Occipital condyle fracture; Outcome

\section{Introduction}

Occipital condyle fractures (OCFs) are rare injuries [1-4]. So far, the detection rate in trauma patients in a centre of the highest care level (level 1 trauma centre) has been mainly based on estimates depending on inclusion criteria [3]. Furthermore, these injuries are principally not visible in conventional X-ray of the cervical spine and require confirmation or exclusion using computed tomography (CT) as a routine measure [2]. This explains why only 20 cases of OCFs had been documented until 1988 [5], while as much as 116 cases were described in the literature between 1978 and 2002 when CT imaging became widely established for diagnosis [1]. However, in an analysis of 47 OCF publications from a period of 35 years, no evidence-based guidelines for OCF therapy have been established, since study populations were too small, and comparative studies were missing [5]. Even the most common OCF classification due to Anderson and Montesano [6] has been based on only 6 retrospective patients, with a further modified classification by Tuli et al. [7] showing only 3 own retrospective cases. The small number of cases explains the variety of different treatment recommendations without any evidence, ranging from conservative therapy by immobilisation through to surgical occipito-cervical fusion [1-7]. Therefore, we decided to conduct a long-term, prospective study, in order to collect valid data about this principally rare injury. Clinical and radiological follow-up results at one year post trauma have already been published [8].

For the first time, the results of the conducted CT studies of fracture morphology are now published with this report, because comprehensive radiological data have also been missing up to now, due to the scarcity of this injury.

\section{Materials and Methods}

All CT scans of the occipital condyles were made in a prospective study in the trauma centre of the University Hospital of Regensburg between $1^{\text {st }}$ Jan 2005 and $31^{\text {st }}$ Dec 2009. The inclusion criterion with all of these cases was a traumatic anamnesis for the detection or exclusion of a cerebral and/or cervical injury, according to the ATLS (Advanced Trauma Life Support) guidelines.

For this, polytrauma CT scans and cervical and cerebral CT scans were reviewed. CT scans were made using a Somatom Sensation 16 system (Siemens, Erlangen, Germany) at a tube voltage of $120 \mathrm{kV}$. Pitch was 0.9 , detector collimation was $0.75 \mathrm{~mm}$. When a condyle fracture had been suspected, an additional skullbase reconstruction using bone kernels B60 was made. The thickness of the axial slice was $1 \mathrm{~mm}$; moreover, sagittal and coronary MPR reconstructions in $2 \mathrm{~mm}$ slices were calculated. Imaging diagnostics comprised polytrauma CT scans (film thickness 5 to $0.75 \mathrm{~mm}$ ), cerebral CT scans (film thickness 3 auf $0.75 \mathrm{~mm}$ ), and cervical CT scans (film thickness 2 auf $0.75 \mathrm{~mm}$ ). If an OCF had been detected, coronary and sagittal reconstructions of the $\mathrm{C} 0-\mathrm{C} 2$ region were made in addition to the axial slices.

All CT scans were documented prospectively. If an OCF was detected (interdisciplinary by radiologists and trauma surgeons), empirical data and the further clinical course of the patient, including injury pattern, were recorded in an additional database as follows: Sex, age, side of localisation, cause of accident, related injuries, initial Glasgow Coma Scale (GCS) rating, and Injury Severity Score (ISS). The type of fracture of detected OCFs was rated in accordance with the Anderson and Montesano classification [6].

*Corresponding author: Mueller Franz, Clinic for Trauma and Orthopaedic Surgery Barmherzige Brüder, Prüfeninger Str. 86, 93049 Regensburg, Germany, Tel: 0049 941-369-2251; Fax: 0941-369-2255; E-mail: franz.mueller@barmherzige-regensburg. de

Received October 14, 2014; Accepted November 21, 2014; Published November 24, 2014

Citation: Franz M, Michael R, Holger G, Bernd F (2014) CT-Based Diagnosis of Occipital Condyle Fractures. A Prospective Evaluation With Reassessment of Fracture Morphology. J Trauma Treat 4: 225. doi:10.4172/2167-1222.1000225

Copyright: (c) 2014 Franz M. This is an open-access article distributed under the terms of the Creative Commons Attribution License, which permits unrestricted use, distribution, and reproduction in any medium, provided the original author and source are credited. 
Citation: Franz M, Michael R, Holger G, Bernd F (2014) CT-Based Diagnosis of Occipital Condyle Fractures. A Prospective Evaluation With Reassessment of Fracture Morphology. J Trauma Treat 4: 225. doi:10.4172/2167-1222.1000225

Irrespective of the fracture type, all patients with confirmed OCFs were receiving a 6-week non-surgical therapy using a hard cervical collar.

12 months post trauma, the first and second author performed a prospective follow-up, for which patients were contacted by telephone or in writing for an outpatient visit. At this occasion, the clinical followup started with a survey using the SF-36 [9] and the Neck Disability Index [10] questionnaires.

For confirming fracture healing, another CT scan of the occipitocervical region was made and compared with scans that had been made at the time of the trauma. At the end of the study, a secondary analysis of fracture morphology (as shown in CT scans) was conducted with regard to possible courses and differences.

The study design were approved by the local ethics committee and informed consent was obtained from each patient.

\section{Statistics}

The SPSS software PASW Statistics 17.0 for Windows was used for statistical data analyses. The Chi square test was used for testing independence, and the non-parametric Mann-Whitney $U$ test was used for significance testing. A significance level of $\leq 0.05$ was used for all tests.

\section{Results}

During the treatment period, a total of 2,616 CT scans were made. A total of 31 patients and 35 OCFs, respectively, were diagnosed, of which 27 patients had unilateral and 4 had bilateral OCFs. The left condyle was fractured in 16 cases, the right condyle in 19 cases.

Thus, the overall OCF incidence was $1.19 \%$, based on the total of 2,616 performed CT scans. Moreover, 3 of 31 patients (9.7\%) were also diagnosed with Atlanto-Occipital Dislocation (AOD).

Gender distribution showed 20 male and 11 female patients, median age was 38.3 years (range: $15-88$ years, $S D \pm 16.35$ ), and in most of the cases the injuries were caused by high speed trauma due to traffic accident. Initial GCS was 11.8 (SD: \pm 4.68 ), and the mean ISS - as an indicator for injury severity - was 25.8 (SD \pm 14.38 ). There were no cases of isolated OCFs. Related intracerebral injuries (ICB, SAB, SDH) were found in 14 of 31 patients (45.16\%). 5 of 31 patients (16.13\%) deceased due to the severity of their intracerebral injuries after a median period of 4 days (range: 0-31 days) post trauma.

None of the fatalities was caused by OCFs. Nevertheless, overall mortality with confirmed OCF was $16.1 \%$. Mortality with unilateral OCF - without additional AOD - was $8.3 \%$ ( 2 of 24 patients). Mortality with confirmed bilateral OCF was $25 \%$ ( 1 of 4 patients), and none of these patients had an additional AOD. Two of these four patients survived the trauma but have been suffering from tetraplegia. An additional AOD was found in 3 of 31 patients (9.7\%): Two patients deceased, and one patient survived after surgical occipito-cervical stabilisation but was wheelchair-ridden and suffering from tetraplegia.

According to the classification of Anderson and Montesano [6], there were 27 cases of unilateral OCFs and 4 cases of bilateral OCFs splitting into a total of 23 type 1, 5 type 2, and 7 type 3 injuries. Statistical analysis showed no significant differences between the three types of fractures with regard to initial GCS, ISS, mortality, or functional outcome.

Irrespective of this classification, analyses of the coronar reconstructions found that 17 patients (19 condyles) showed a vertical fracture line (Figures 1a-1c) with articular surface involvement, and 14 patients (16 condyles) showed a horizontal fracture line (Figures 2a-2c) without involvement of the articular surface. In 17 patients with vertical fracture lines, median GCS was 15 and median ISS was 17 . None of the patients with vertical fracture lines died; thus, mortality rate was $0 \%$. In patients with horizontal fracture lines, median GCS was 8.5 and median ISS was 31.5. Five of 14 patients with horizontal fracture lines died, and the mortality rate was $35.7 \%$. In summary, there were clear - but non-significant - differences between cases with vertical versus horizontal fracture lines with regard to initial GCS and ISS scores. However, mortality was significantly higher in the latter cases.

Prospectively, 22 of 31 patients (71\%) were followed up radiologically and clinically 1 year after trauma. Five patients $(16.13 \%)$

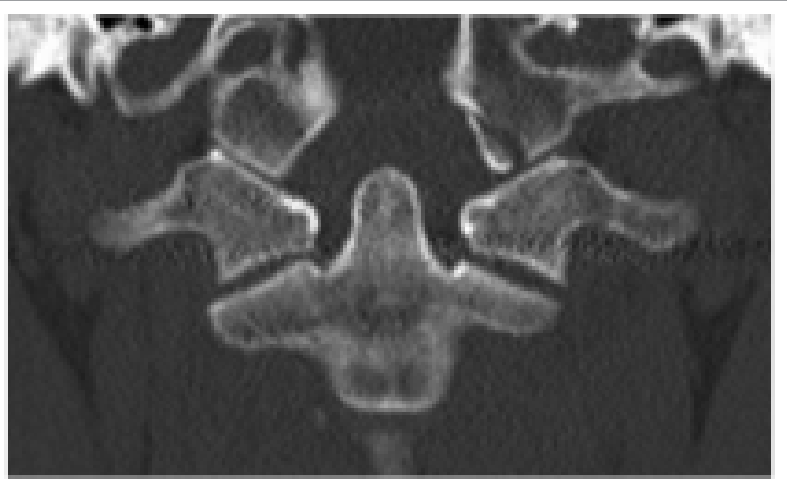

Figure 1a: CT with coronar reconstruction: Patient with OCF on the left side with vertical fracture line.

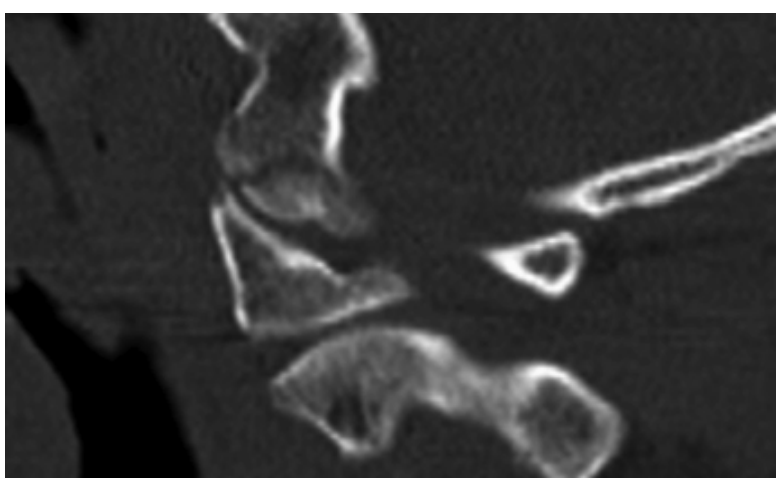

Figure 1b: Same patient; sagittal reconstruction

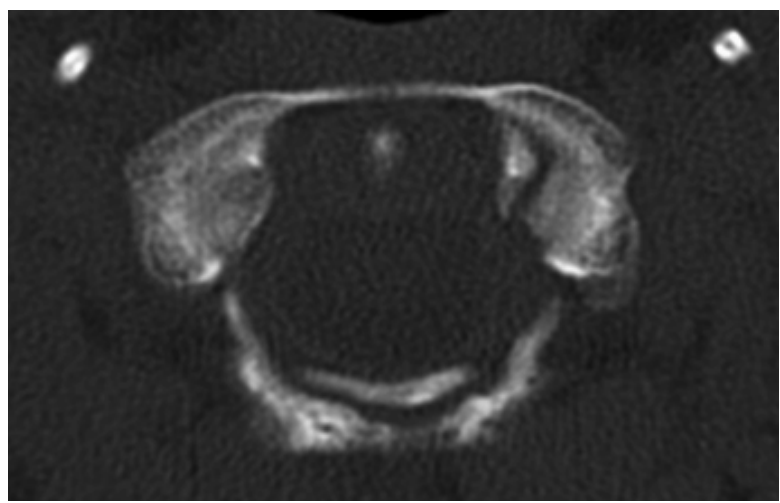

Figure 1c: Same patient; axial reconstruction. 
Citation: Franz M, Michael R, Holger G, Bernd F (2014) CT-Based Diagnosis of Occipital Condyle Fractures. A Prospective Evaluation With Reassessment of Fracture Morphology. J Trauma Treat 4: 225. doi:10.4172/2167-1222.1000225

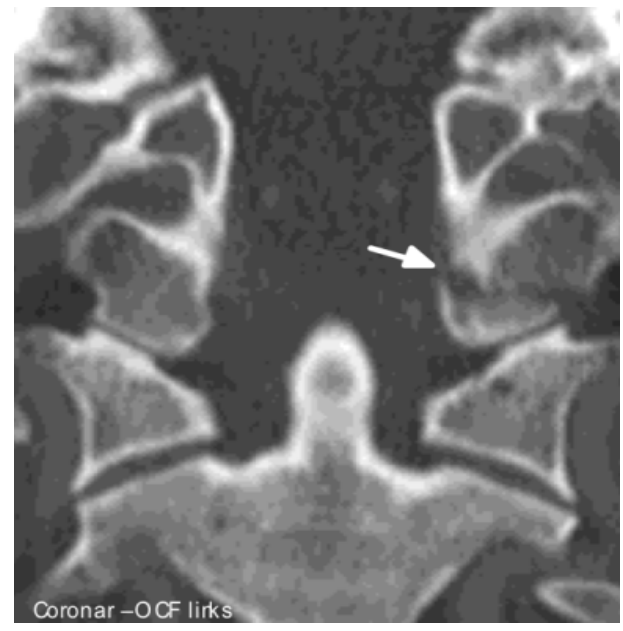

Figure 2a: CT with coronar reconstruction: Patient with OCF of the left side with horizontal fracture line.

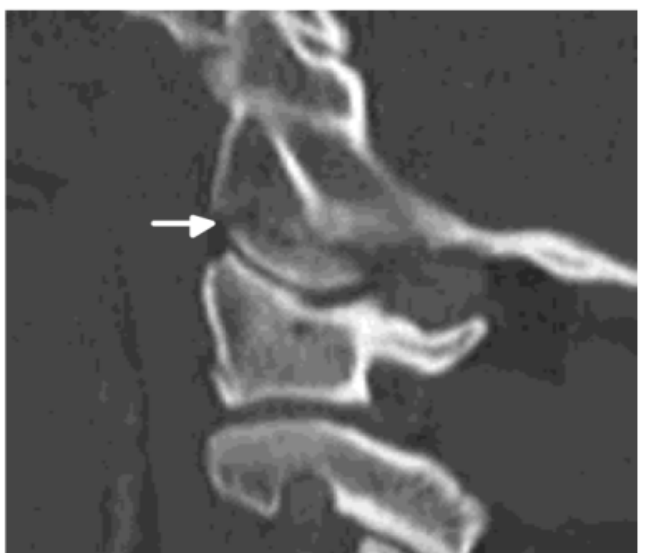

Figure 2b: Same patient; sagittal reconstruction.

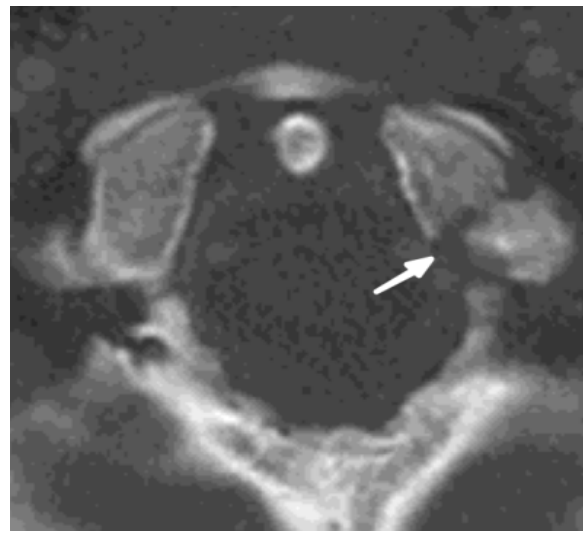

Figure 2c: Same patient; axial reconstruction.

had died, 4 patients refused follow-up, of which 2 were bedridden and had tetraplegia. Only 1 patient (OCF with AOD) out of 3 patients with tetraplegia was followed up clinically and radiologically. In 21 of 22 patients, CT scans showed bony consolidation of the fractures, and there was only one case showing an unchanged dislocated fragment Thus, neither secondary fragment dislocation nor pseudarthrosis was observed in any of the cases. Furthermore, no signs of arthrosis were found in any of the cases at the time of follow-up.

Clinical evaluation of the Neck Disability Index [10] showed a median score of 10.5 points (SD: \pm 8.3 ), equivalent to a disability degree of $21 \%$ (SD: $\pm 16.6 \%$ ) and to slight limitation, respectively. The SF-36 questionnaire [9] showed that the quality of life was significantly reduced in all domains 1 year after trauma, which however was due to the overall trauma pattern rather than due to the OCF.

Clinical and radiological follow-up comprised 8 patients with horizontal and 16 patients with vertical fracture lines, and all of the 3 tetraplegic patients had horizontal fracture lines. For the NDI analysis, the worst result was assigned to both of the tetraplegic patients who were not examined. Analysis of the patients with horizontal fracture lines (including both tetraplegic patients) resulted in a median NDI of $45 \%$ (SD: \pm 33.29 ), equivalent to severe disability, and the median total SF-36 score was 71.65 points ( $\mathrm{min} .35$ to max. 150 points).

NDI in patients with vertical fracture lines was 17\% (SD: \pm 15.43 ) equivalent to a minor degree of disability, and the median total SF-36 score was 114.55 points (SD: \pm 22.13 ). The clinical difference between NDI and SF-36 scores was significant. Since patients with horizontal fracture lines and those with associated intracerebral injury showed a significantly worse outcome, a possibly relationship has been suspected. The Fisher's exact test resulted in a p-value of 0.012 and confirmed the hypothesis of a significant relationship between horizontal fracture lines and related intracerebral injuries.

\section{Discussion}

First of all, it must be outlined that most of the clinical data of this long-term prospective study have already been published [8]. However, no comprehensive analysis of the CT scans used for diagnosis has been performed, since this procedure has not been acknowledged adequately on an international level due to the small number of cases.

This study is the first to prospectively collect OCF data in a trauma centre over a period of more than six years (five years of data collection, and one year of post-trauma follow-up) to summarise a larger number of cases. The incidence of this injury in relation to the performed CT scans was $1.19 \%$. Up to now, other studies provided only estimates, depending on the inclusion criteria, with a significantly lower incidence $[2,4]$. Our data indicate that OCFs must be expected in at least 1 of 100 patients, if - after relevant injuries and in accordance with the ATLS guidelines - diagnostic CT scans of the occipital condyles are indicated.

Tuli et al. [7] report an approximately equal incidence (1 of 93 patients), but these data were collected retrospectively with a smaller number of cases. It must be emphasised that it is principally not possible to detect OCFs with conventional X-ray imaging $[7,11]$. If such injuries are suspected in children, an MRI scan can be performed for OCF detection, in order to prevent radiation exposure [12]. However, we still consider computed tomography to be the so-called "Gold standard" for the confirmation/exclusion of OCFs.

Altogether, a mortality rate of $16.1 \%$ was found with unilateral OCFs. If no AOD had been associated, this rate declined to $8.3 \%$ (8 of 24 patients). Thus, these results are in the range of other study data $[2,4]$. Bilateral condyle fractures were particularly observed in lethal injury patterns. [11]. In such cases, much higher kinetic forces must be expected, leading to high ISS scores. Even in our patient population - with only 4 patients - a significantly higher morbidity and mortality for bilateral fractures was shown when compared to unilateral 
Citation: Franz M, Michael R, Holger G, Bernd F (2014) CT-Based Diagnosis of Occipital Condyle Fractures. A Prospective Evaluation With Reassessment of Fracture Morphology. J Trauma Treat 4: 225. doi:10.4172/2167-1222.1000225

OCFs without AOD. In our study, OCFs with additional AOD were diagnosed in 3 patients $(9.7 \%)$. Mortality is significantly increased with this very rarely observed subaxial combination injury when compared to unilateral or bilateral OCFs without AOD and it was almost $67 \%$ in our study, also with a small number of cases.

Anderson and Montesano [6] described 3 types of fractures. And although it must be emphasised that this 3-type classification is only based on 6 retrospective cases, it has not been modified since then and it is still used for classification and reference, even in current studies and reviews $[2,13]$. It was already mentioned in other studies that the classification in accordance with Anderson and Montesano [6] might be difficult, since diagnostic findings are not always clearly attributable to type 1,2 or 3 injuries [2,4]. This is also reflected by the variety of observed frequency rates: Malham et al. [3] and Maserati et al. [4] diagnosed less than $30 \%$ of type 3 injuries in their studies, while Hansen et al. [2] diagnosed 75\% of type 3 injuries. We can also confirm such classification difficulties, and our classification may also have a lower inter- or intra-observer reliability, particularly with regard to type 1 and type 3 injuries. However, investigation of validity was not an objective of the study. On the other hand, the statistical analysis did not show significant differences between these 3 types of fractures with regard to trauma severity (ISS), radiological and clinical outcome, and mortality, when all patients received non-surgical immobilisation treatment using a cervical collar for 6 weeks. Therefore, we think that this classification can be disregarded and it does not have clinical implications.

Surprisingly, comprehensive analysis of the fracture morphology at the end of the study using CT scans showed two lines of fractures that have not been documented in this manner in the literature before. In coronar reconstructions, two different fracture lines have been clearly identified: In 17 patients (i.e. 19 occipital condyles) a vertical fracture line was observed extending into the $\mathrm{C} 0 / \mathrm{C} 1$ joint; and in 14 patients (i.e.16 condyles) a horizontal fracture line was observed in the condyle region without involvement of the articular surface.

On the other hand, we have not yet seen a fracture morphology in our patient population with possible occipital fragmented fractures as it has been described (at least in a graphical manner) by Anderson/ Montesano [5] as type 1 injury. This may in fact complicate the distinction between horizontal versus vertical fracture lines or make it even impossible.

The statistical analysis of both fracture lines showed relevant principal aspects with significant relations: Both mortality and clinical outcome were significantly worse in cases of horizontal fracture lines, although not based on the fracture itself but in relation with related intracerebral injuries (i.e. SAB or ICB).

This may lead to suspecting and possibly postulating that shear and/or rotational forces are primarily causing horizontal fracture lines - in relation with the severity of the trauma altogether - while vertical fracture lines are mainly caused by compression forces.

Finally, it must be emphasised once again that uni- or bilateral OCFs represent benign injuries - irrespective of the shape of fracture - and achieve bony consolidation within one year post trauma. Nevertheless, fracture morphology can serve as an indicator for the outcome, which is determined by related cerebral injuries.

\section{Conclusion}

The analysis of fracture lines in coronar CT scans provides information regarding the clinical course. Horizontal fractures - when compared to vertical fractures - are associated with higher morbidity and mortality, and with a significantly worse outcome. Nevertheless, uni- or bilateral OCFs per se represent benign injuries - irrespective of the shape of fracture - and achieve bony consolidation within one year post trauma. Quality of life is compromised by comorbidities, particularly by related intracerebral injuries, rather than by the OCF.

\section{References}

1. Caroli E, Rocchi G, Orlando ER, Delfini R (2005) Occipital condyle fractures: report of five cases and literature review. Eur Spine J 14: 487-492.

2. Hanson JA, Deliganis AV, Baxter AB, Cohen WA, Linnau KF, et al. (2002) Radiologic and clinical spectrum of occipital condyle fractures: retrospective review of 107 consecutive fractures in 95 patients. AJR Am J Roentgenol 178 1261-1268.

3. Malham GM, Ackland HM, Jones R, Williamson OD, Varma DK (2009) Occipita condyle fractures: incidence and clinical follow-up at a level 1 trauma centre. Emerg Radiol 16: 291-297.

4. Maserati MB, Stephens B, Zohny Z, Lee JY, Kanter AS, et al. (2009) Occipital condyle fractures: clinical decision rule and surgical management. J Neurosurg Spine 11: 388-395

5. Hadley MN, Walters BC, Grabb PA, Oyesiku NM, Przybylski GJ, et al. (2002) Occipital condyle fractures. Neurosurgery 50: 114-119.

6. Anderson PA, Montesano PX (1988) Morphology and treatment of occipital condyle fractures. Spine (Phila Pa 1976) 13: 731-736.

7. Tuli S, Tator $\mathrm{CH}$, Fehlings MG, Mackay M (1997) Occipital condyle fractures Neurosurgery 41: 368-376.

8. Mueller FJ, Fuechtmeier B, Kinner B, Rosskopf M, Neumann C, et al. (2012) Occipital condyle fractures. Prospective follow-up of 31 cases within 5 years at a level 1 trauma centre. Eur Spine J 21: 289-294.

9. Bullinger M, Kirchberger I, Ware JE (1995) The German SF-36 health survey translation and psychometric testing of a generic instrument for the assessment of health-related quality of life. Journal of Public Health 3: 21-36.

10. Vernon H, Mior S (1991) The Neck Disability Index: a study of reliability and validity. J Manipulative Physiol Ther 14: 409-415.

11. Leone A, Cerase A, Colosimo C, Lauro L, Puca A, et al. (2000) Occipital condylar fractures: a review. Radiology 216: 635-644.

12. Kapapa T, Tschan CA, König K, Schlesinger A, Haubitz B, et al. (2006) Fracture of the occipital condyle caused by minor trauma in child. J Pediatr Surg 41: 1774-1776.

13. Maddox JJ, Rodriguez-Feo JA 3rd, Maddox GE, Gullung G, McGwin G, et al. (2012) Nonoperative treatment of occipital condyle fractures: an outcomes review of 32 fractures. Spine (Phila Pa 1976) 37: E964-968. 